\title{
Greek Basidiomycete Wild Strains for the Production of Bioactive Compounds and Enzymes with Applications in Cosmetic and Biocatalysis Industries ${ }^{\dagger}$
}

\author{
Anastasia Zerva ${ }^{1}$, Nikolaos Tsafantakis ${ }^{2}$ and Evangelos Topakas ${ }^{1, *}$ \\ 1 Industrial Biotechnology \& Biocatalysis Group, School of Chemical Engineering, National Technical \\ University of Athens, 9 Iroon Polytechniou Str., Zografou Campus, Athens 15780, Greece; \\ anazer@chemeng.ntua.gr \\ 2 Department of Pharmacognosy and Natural Products Chemistry, Faculty of Pharmacy, National and \\ Kapodistrian University of Athens, Athens, 15771, Greece; ntsafantakis@pharm.uoa.gr \\ * Correspondence: vtopakas@chemeng.ntua.gr; Tel.: +30-210-772-3264 \\ + Presented at the 1st International Electronic Conference on Catalysis Sciences, 10-30 November 2020; \\ Available online: https://sciforum.net/conference/ECCS2020.
}

Published: 10 November 2020

\begin{abstract}
White-rot basidiomycetes are the only microorganisms able to produce both hydrolytic (cellulases and hemicellulases) and oxidative (ligninolytic) enzymes for attacking (hemi)cellulose and lignin, while they also produce secondary metabolites with important applications as cosmeceuticals. In the present work, three wild strains of Basidiomycete fungi (Pleurotus citrinopileatus, Abortiporus biennis and Ganoderma lucidum) were grown in agroindustrial residues, such as olive oil mill wastewater and corn cob. The cultures were examined in regard to the production of biotechnologically relevant enzymes and bioactive compounds. All strains were found to be preferential lignin degraders. Bioinformatic analyses were performed on the putative proteome of the strains P. citrinopileatus and A. biennis, focusing on CAZymes with biotechnological relevance, and the results were compared to the data from the enzyme activities in the culture supernatants. The antiaging proprieties of fungal biomasses have been evaluated in relation to the different culture media. P. citrinopileatus showed the highest skin whitening activity, while A. biennis the highest DPPH scavenging potential. For all strains, corn cobs was the most appropriate carbon source for the production of bioactive small molecules with anti-aging activity. Overall, all strains showed promising antiaging properties and strong production of promising oxidative enzymes for biomass conversion applications.
\end{abstract}

Keywords: mushrooms; basidiomycetes; novel enzymes; biocatalysts; natural bioactive compounds; secondary metabolites; laccases; LPMOs

\section{Introduction}

White-rot basidiomycetes are the only microorganisms producing hydrolytic (cellulases and hemicellulases) and oxidative (ligninolytic) enzymes for attacking both cellulose/hemicellulose and lignin. In addition, they produce secondary metabolites with important applications in the cosmetics industry. Especially ligninolytic enzymes can be applied in numerous fields of industry, e.g. fuel, food, agriculture, paper, textile and cosmetics industries. Lignin is highly recalcitrant to microbial degradation [1]. Certain white-rot basidiomycetes in particular, are able to degrade lignin, cellulose and hemicellulose concomitantly, while others attack lignin selectively by secreting enzymes, collectively named "ligninases" [2]. Ligninases can be divided in separate classes, namely phenol oxidases (laccases) and heme peroxidases. Apart from ligninolytic enzymes, white-rot fungi also secrete cellulases, xylanases and other hemicellulases. These enzymes act on long-chain polysaccharides, mainly cellulose and hemicellulose, resulting in the hydrolysis of these materials to 
their monomer sugars. The substrates used for fungal growth are usually agricultural or agroindustrial residues of low economic value and in many cases with toxic effects (e.g. olive-mill wastes). Recent studies revealed that the composition of cultivation substrates can significantly affect nutritional properties of mushrooms [3] or their antioxidant activity. Unfortunately, a huge gap still exists in our knowledge regarding the effect that cultivation substrates could exert on the production of bioactive metabolites, and/or ligninolytic enzymes by edible/medicinal basidiomycetes.

In the cosmetic sector, secondary metabolites can be found in several antiaging formulations due to their anti-oxidant and anti-tyrosinase proprieties. Most of tyrosinase inhibitors derive from natural sources including various microorganisms [4]. Tyrosinase is the key enzyme of melanin biosynthesis in microorganisms, plants, and animals. Tyrosinase inhibitors are mainly used for the treatment and prevention of hyperpigmentation in the epidermal layers of the human skin. Despite that the clinical and industrial demands for tyrosinase inhibitors increase, nowdays there are very few compounds certified for clinical use [5]. Thus, there is a strong need for new anti-tyrosinase agents.

In the present work, wild strains of edible/medicinal basidiomycetes isolated from diverse habitats of Greek territory were evaluated for the production of enzymes with cellulolytic and ligninolytic activity and bioactive small molecules with anti-aging activity.

\section{Methods}

\subsection{Microorganisms and Culture Procedures}

The P. citrinopileatus LGAM 28684, Abortiporus biennis LGAM 436 and Ganoderma resinaceum LGAM 334 strains used for this study, were obtained from the fungal culture collection of the Laboratory of General and Agricultural Microbiology (Agricultural University of Athens). OMWW was obtained from an olive oil mill with a three-phase decanter in Kalamata (Peloponnese, S.W. Greece) and maintained at $-20{ }^{\circ} \mathrm{C}$ [6]. Prior to use, $\mathrm{pH}$ was adjusted to 6 with $3 \mathrm{~N} \mathrm{NaOH}$, and the suspended solids were removed. For the preparation of liquid cultures, OMWW was diluted at a final concentration of $50 \%(\mathrm{v} / \mathrm{v})$ with $100 \mathrm{mM}$ phosphate buffer $\mathrm{pH} 6$ to a final volume of $50 \mathrm{~mL}$. Alternatively, corn cobs (CC) or Avicel were added at a final concentration of $30 \mathrm{~g} \mathrm{~L}^{-1}$ as carbon source. Corn steep liquor was added as nitrogen source at a final concentration of $3 \mathrm{~g} \mathrm{~L}^{-1}$. After inoculation, liquid cultures were incubated at $26^{\circ} \mathrm{C}, 100 \mathrm{rpm}$, unless otherwise stated. Samples were taken at selected time intervals, centrifuged (3000 rpm, $10 \mathrm{~min})$, and the supernatant was used for analysis and determination of enzyme activities.

For the evaluation of the antioxidant and skin whitening activity, biomasses were separated from culture supernatants by filtration using whatman filter paper No 4 and lyophilized. $0.4 \mathrm{~g}$ of each dried biomass was subjected to ultrasound-assisted extraction for $40 \mathrm{~min}$ at $25{ }^{\circ} \mathrm{C}$ using $10 \mathrm{~mL}$ of ethyl acetate. Each sample was extracted 3 times, supernatants were centrifuged ( $4000 \mathrm{rpm}, 3 \mathrm{~min}$ ), combined and dried under reduced pressure using a centrifugal vacuum concentrator. Extracts were immediately stored at $-20^{\circ} \mathrm{C}$ prior to analysis.

\subsection{Enzyme Assays and Analyses}

Laccase activity was determined as described in [7]. Cellulase and xylanase activities were measured as described in [8], using carboxymethyl cellulose, avicel or birchwood xylan as substrates. The determination of reducing sugars was performed with the DNS method [9]. For the detection of LPMO activity, $200 \mu \mathrm{L}$ of culture supernatant were added to $500 \mu \mathrm{L}$ of phosphoric acid-swollen cellulose (PASC) $2 \%(\mathrm{w} / \mathrm{v})$ in $50 \mathrm{mM}$ acetate buffer $\mathrm{pH}$ 5.2. Cysteine was added as electron donor at a final concentration of $1 \mathrm{mM}$. The mixture was incubated for $16 \mathrm{~h} \mathrm{in} 45^{\circ} \mathrm{C}$ and $1000 \mathrm{rpm}$. The reaction was terminated by boiling, and the supernatant was analyzed for oxidized sugars with HPAEC-PAD, as described previously [10].

Total phenols content was determined as previously described [7] and it was expressed in ppm of gallic acid equivalents. OMWW decolorization was calculated by measuring the absorbance at 525 $\mathrm{nm}$, as previously described [7]. 


\subsection{Bioinformatics Analyses}

Automated CAZyme annotation was performed to the predicted protein sequences of $P$. citrinopileatus and A. biennis, with the dbCAN2 meta server, employing the HMMER, DIAMOND and HotPep tools [11].

\subsection{Free Radical Scavenging (DPPH) and Tyrosinase Inhibition Assay}

The protocol based on [12] was used as follows: A stock solution of $0.314 \mathrm{mM}$ DPPH in ethanol was prepared. Stock solutions of samples in DMSO $\left(4 \mathrm{mg} \mathrm{mL}^{-1}\right)$ were prepared. $10 \mu \mathrm{l}$ of extract in DMSO and $190 \mu \mathrm{l}$ of DPPH solution were mixed and incubated for $30 \mathrm{~min}$ at $25^{\circ} \mathrm{C}$ in the dark. Absorbance was measured at $517 \mathrm{~nm}$. Gallic acid was used as positive control. The percentage DPPH scavenging was estimated by the following equation: $[(A-B)-(C-D)] /(A-B) \times 100$, where $A$ : Control (w/o sample), B: Blank (w/o sample, w/o DPPH), C: sample, D: Blank sample (w/o DPPH). Extracts were tested at $200 \mu \mathrm{g} \mathrm{mL}^{-1}$ and $100 \mu \mathrm{g} \mathrm{mL}^{-1}$ (final concentration).

The anti-tyrosinase ability of the extracts was evaluated as described in [13]. Extracts were dissolved in DMSO and diluted in phosphate buffer 1/15 M, pH 6.8. In 96-well plates, $80 \mu \mathrm{l}$ of phosphate buffer $1 / 15 \mathrm{M}, \mathrm{pH} 6.8,40 \mu \mathrm{l}$ of sample and $40 \mu \mathrm{l}$ mushroom tyrosinase ( 92 Units $\mathrm{mL}^{-1}$ ) were mixed. The contents of each well were incubated for $10 \mathrm{~min}$ at $25^{\circ} \mathrm{C}$, before $40 \mu \mathrm{l}$ of $2.5 \mathrm{mM} \mathrm{L}$ DOPA were added. After incubation at $25{ }^{\circ} \mathrm{C}$ for $5 \mathrm{~min}$, the absorbance at $475 \mathrm{~nm}$ was measured using a TECAN plate reader. Kojic acid was used as positive control. The percentage inhibition of the tyrosinase activity was calculated by the following equation: $[(A-B)-(C-D)] /(A-B) \times 100$, where A: Control (w/o sample), B: Blank (w/o sample, w/o tyrosinase), C: Sample, D: Blank sample (w/o tyrosinase). Extracts and fractions were tested at $300 \mu \mathrm{g} \mathrm{mL}^{-1}$ and $100 \mu \mathrm{g} \mathrm{mL} \mathrm{L}^{-1}$ (final concentration in the well).

\section{Results and Discussion}

In our first steps towards the potential exploitation of wild strains of edible/medicinal basidiomycetes in cosmetic and biocatalysis industries, we prepared liquid cultures of A. biennis, P. citrinopileatus and G. resinaceum using different lignocellulosic waste materials as carbon sources. The fungal biomasses were extracted and evaluated for their anti-oxidant and anti-tyrosinase acitivities while culture supernatants were evaluated for the production of enzymes with cellulolytic and ligninolytic activity, respectively. P. citrinopileatus showed the most interesting skin whitening activity, followed by G. resinaceum, and by A. biennis (Table 1). For all strains, CC was the most appropriate carbon source for maximizing the potential production of bioactive small molecules with anti-aging activity. The use of OMWW as carbon source sligtly descreased the anti-tyrosinase potential of G. resinaceum and of A. biennis, while for P. citrinopileatus resulted in the complete loss of bioactivity (Table 1). The DPPH scavenging potential of the genarated extracts also drastically decreased with OMWW as carbon source, conluding that CC is the most appropriate carbon source for the production of anti-oxidant and anti-tyrosinase compounds from the aforementioned strains (Table 1).

Table 1. Anti-oxidant and anti-tyrosinase evaluation of fungal biomasses.

\begin{tabular}{|c|c|c|c|c|c|}
\hline \multirow{2}{*}{\multicolumn{2}{|c|}{ Carbon source*Fungal extracts }} & \multicolumn{4}{|c|}{$\%$ Tyrosinase Inhibition $( \pm$ SD) $\%$ DPPH Scavenging $( \pm$ SD) } \\
\hline & & $300 \mu \mathrm{g} \mathrm{mL}-1$ & $100 \mu \mathrm{g} \mathrm{mL}^{-1}$ & $200 \mu \mathrm{g} \mathrm{mL^{-1 }}$ & $100 \mu \mathrm{g} \mathrm{mL} \mathrm{L}^{-1}$ \\
\hline \multirow{3}{*}{ OOMW } & Abi & $23.22 \pm 1.47$ & $3.61 \pm 3.16$ & $0.46 \pm 0.58$ & $-1.19 \pm 1.00$ \\
\hline & Pci & $3.42 \pm 0.83$ & $7.39 \pm 3.73$ & $0.57 \pm 2.40$ & $-1.34 \pm 0.69$ \\
\hline & Gre & $22.13 \pm 0.84$ & $6.60 \pm 2.39$ & $0.58 \pm 0.28$ & $-0.23 \pm 1.43$ \\
\hline \multirow{3}{*}{$\mathrm{CC}$} & Abi & $25.20 \pm 1.85$ & $4.76 \pm 2.56$ & $24.01 \pm 0.14$ & $12.04 \pm 0.25$ \\
\hline & Pci & $32.00 \pm 0.92$ & $27.13 \pm 0.62$ & $17.57 \pm 0.41$ & $10.40 \pm 1.44$ \\
\hline & Gre & $27.25 \pm 0.96$ & $12.24 \pm 0.20$ & $16.71 \pm 0.42$ & $6.69 \pm 0.62$ \\
\hline
\end{tabular}

${ }^{*}$ Cultivations were carried out in duplicates. Abi: A. biennis, Pci: P. citrinopileatus, Gre: G. resinaceum. 
The enzymatic variability of the studied strains in regard to lignocellulose degradation was assessed by assembling the predicted CAZymes of the available genome sequences using state-of theart annotation tools, such as dbCAN2 meta server. The annotation was performed for the publicly available genome sequences of P. citrinopileatus and A. biennis, as shown in Table 2.

Table 2. Predicted protein sequences present in the A. biennis and P. citrinopileatus genome, relative to lignocellulose degradation.

\begin{tabular}{|c|c|c|c|c|}
\hline \multicolumn{2}{|r|}{ Specific Activity } & \multirow[t]{2}{*}{ CaZy Modules } & \multicolumn{2}{|c|}{ No of Predicted Sequences } \\
\hline & & & A. biennis & P. citrinopileatus \\
\hline \multirow[t]{3}{*}{ Cellulases } & Endoglucanases & GH 5, 7, 12, 45 & 14 & 17 \\
\hline & Cellobiohydrolases & GH 6,7 & 4 & 10 \\
\hline & $\beta$-glucosidases & $\mathrm{GH} 1,3$ & 2 & 5 \\
\hline \multirow[t]{2}{*}{ Xylanases } & Endoxylanases & GH 10, 11 & 6 & 15 \\
\hline & $\beta$-xylosidases & GH 3, 43 & 4 & 7 \\
\hline \multirow[t]{4}{*}{ Oxidases } & Laccases & AA 1 & 8 & 16 \\
\hline & Peroxidases & AA 2 & 12 & 11 \\
\hline & $\begin{array}{l}\text { Alcohol oxidases and } \\
\text { cellobiose dehydrogenases }\end{array}$ & AA 3 & 3 & 16 \\
\hline & $\begin{array}{l}\text { Lytic polysaccharide } \\
\text { monooxygenases }\end{array}$ & AA $9,10,11,13,14$ & 12 & 28 \\
\hline
\end{tabular}

The genomes of both strains include all the necessary enzymatic activities for the breakdown of major lignocellulose components, and in most cases in multiple gene copies. P. citrinopileatus was found to contain more copies of oxidative enzymes and endoxylanases than A. biennis. Especially for laccases, Pleurotus species are known to be potent producers [14], and P. citrinopileatus was shown to be no exception. Nonetheless, both strains are shown to possess the biocatalytic potential for complete degradation of lignocellulosic biomass and relative substrates. However, the existence of the required genes does not necessarily lead to efficient induction, expression, and secretion of the respective proteins. For this reason, the aforementioned fungal strains, together with G. resinaceum, which is a strain with unknown genome sequence, were grown in different lignocellulosic materials as carbon sources, and the profile of secreted enzyme activities was explored in reaction supernatants.

All of the studied strains were found able to achieve complete breakdown of OOMW phenolic load. The maximum phenols degradation was observed by $P$. citrinopileatus, reaching up to $94.3 \pm$ $0.9 \%$ together with $86.1 \pm 0.6 \%$ color removal. A. biennis achieved up to $87.5 \pm 1.5 \%$ phenols degradation and up to $71.5 \pm 0.8 \%$ color removal, while for $G$. resinaceum phenols degradation reached $86.0 \pm 2 \%$ and the respective color removal was $74.0 \pm 0.1 \%$. The time profile of laccase production is shown in Figure 1a. Surprisingly, P. citrinopileatus was found to be the least potent laccase producer, while G. resinaceum showed the highest laccase production, up to $2.1 \pm 0.04 \mathrm{U} \mathrm{mL}^{-1}$.
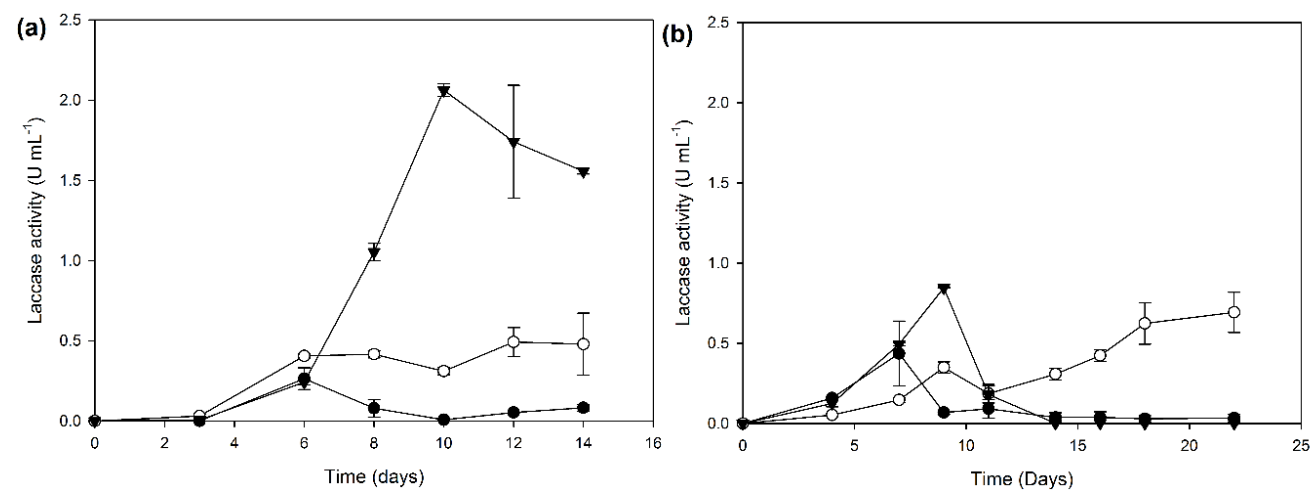

Figure 1. Laccase activity during the growth of P. citrinopileatus (black circles), A. biennis (white circles) and G. resinaceum (black inverted triangles), on OOMW (a) and CC (b). 
The next step was to study the enzyme production during growth on an untreated lignocellulosic substrate, therefore, the three fungal strains were grown in CC, in shaking liquid cultures. Endocellulase, endoxylanase, and laccase activities were monitored during the course of the culture. The results for laccase activity are shown in Figure 1b. Laccase activity for all strains was found to be significantly lower than when grown on OOMW, which is expected due to the low lignin content of the substrate. Again, G. resinaceum was found to be the most potent laccase producer from the three strains. Surprisingly, endocellulase and endoxylanase activities were not detected at any time point during the course of the fungal cultures. This results might be partly explained by the physiology of white-rot basidiomycetes, most of which are preferential lignin degraders [2].

Despite the absence of detectable endocellulase or endoxylanase activity, soluble reducing sugars were detected in the culture supernatants, up to $0.66 \mathrm{mg} \mathrm{mL}^{-1} \pm 0.004$ for $P$. citrinopileatus, 0.59 $\pm 0.04 \mathrm{mg} \mathrm{mL}^{-1}$ for $A$. biennis and $0.41 \pm 0.01 \mathrm{mg} \mathrm{mL}^{-1}$ for $G$. resinaceum, indicating the presence of at least some enzyme activity on biomass polysaccharides. All the above data, together with the presence of several LPMO gene copies in the genomes of $P$. citrinopileatus and A. biennis, support the existence of potent LPMO activity. In order to detect LPMO activity, the three fungal strains were grown in microcrystalline cellulose (Avicel) as sole carbon source. After 15 days of growth, the supernatants were analyzed for LPMO activity. As shown in Figure 2, LPMO activity was detected in the supernatants of all fungal strains, albeit the presence of oxidized sugars [15] is more prominent in P. citrinopileatus samples. Our results confirm the expression of LPMOs from the studied strains for cellulose degradation, however, the exact role and significance of LPMO activity during fungal growth in natural complex substrates is still unknown.

(a)

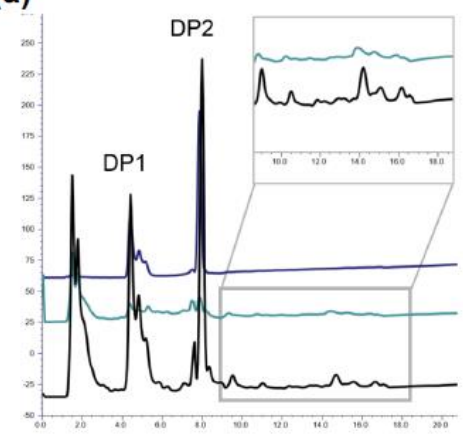

(b)

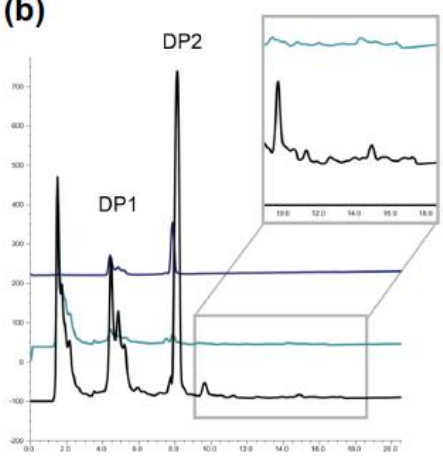

(c)

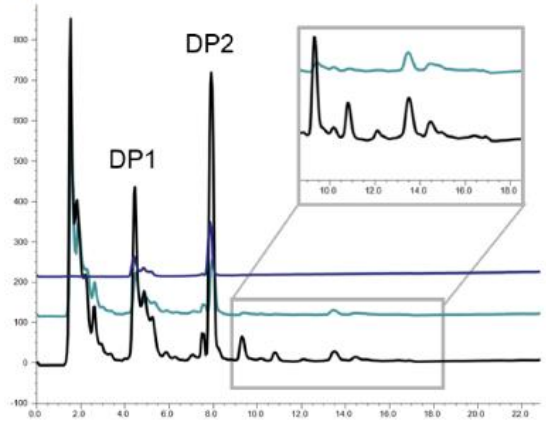

Figure 2. HPAEC-PAD chromatograms for the detection of LPMO activity. (a) P. citrinopileatus, (b) G. resinaceum, (c) A. biennis. Dark blue line: glucose and cellobiose controls, light blue line: reaction control with deactivated enzyme, black line: reaction of crude enzyme with PASC substrate.

\section{Conclusions}

In the present work, a screening study was performed for three native white-rot basidiomycete strains, P. citrinopileatus, A. biennis and G. resinaceum in two different agroindustrial byproducts: OOMW and CC. P. citrinopileatus showed the most interesting skin whitening activity while A. biennis the highest DPPH scavenging potential. For all strains, CC was the most appropriate carbon source for maximizing the production potential of bioactive small molecules. All strains showed significant degradative potential towards OOMW phenols, and by the production of laccase activity. The strains were found to be preferential lignin degraders, similarly to most white-rot fungi. Automated annotation tools revealed that $P$. citrinopileatus and A. biennis contain multiple copies of the necessary genes for complete lignocellulose breakdown. Overall, all strains show promising antiaging properties and significant potential for the discovery of novel enzyme activities.

Author Contributions: Conceptualization, E.T.; methodology, A.Z. and N.T.; investigation, A.Z. and N.T.; writing-original draft preparation, A.Z.; writing-review and editing, A.Z. and N.T.; visualization, A.Z.; supervision, E.T.

Funding: This research is funded in the context of the project "Biotechnological exploitation of wild strains of Greek basidiomycetes for the production of bioactive small molecules and macromolecules with applications in 
cosmetic and biocatalysis industries - BIO2" (MIS 5049430) under the call for proposals "Researchers' support with an emphasis on young researchers- 2nd Cycle". The project is co-financed by Greece and the European Union (European Social Fund- ESF) by the Operational Programme Human Resources Development, Education and Lifelong Learning 2014-2020.

Acknowledgments: Prof. George Zervakis, Agricultural University of Athens, Department of Crop Science, is gratefully acknowledged for the donation of Pleurotus citrinopileatus LGAM 28684, Abortiporus biennis LGAM 436 and Ganoderma resinaceum LGAM 334 strains.

Conflicts of Interest: The authors declare no conflict of interest. The funders had no role in the design of the study; in the collection, analyses, or interpretation of data; in the writing of the manuscript, or in the decision to publish the results.

\section{References}

1. Mussatto, S.I.; Fernandes, M.; Milagres, A.M.F.; Roberto, I.C. Effect of hemicellulose and lignin on enzymatic hydrolysis of cellulose from brewer's spent grain. Enzyme Microb. Technol. 2008, 43, 124-129.

2. Schilling, J.S.; Kaffenberger, J.T.; Held, B.W.; Ortiz, R.; Blanchette, R.A. Using Wood Rot Phenotypes to Illuminate the "Gray" Among Decomposer Fungi. Front. Microbiol. 2020, 11, 1288.

3. Koutrotsios, G.; Larou, E.; Mountzouris, K.C.; Zervakis, G.I. Detoxification of Olive Mill Wastewater and Bioconversion of Olive Crop Residues into High-Value-Added Biomass by the Choice Edible Mushroom Hericium erinaceus. Appl. Biochem. Biotechnol. 2016 180, 195-209.

4. Georgousaki, K.; Tsafantakis, N.; Gumeni, S.; Gonzalez, I.; Mackenzie, T.A.; Reyes, F.; Lambert, C.; Trougakos, I.P.; Genilloud, O.; Fokialakis, N. Screening for tyrosinase inhibitors from actinomycetes; identification of trichostatin derivatives from Streptomyces sp. CA-129531 and scale up production in bioreactor. Bioorganic Med. Chem. Lett. 2020, 30, 126952.

5. Zolghadri, S.; Bahrami, A.; Hassan Khan, M.T.; Munoz-Munoz, J.; Garcia-Molina, F.; Garcia-Canovas, F.; Saboury, A.A. A comprehensive review on tyrosinase inhibitors. J. Enzyme Inhib. Med. Chem. 2019, 34, $279-309$.

6. Ntougias, S.; Gaitis, F.; Katsaris, P.; Skoulika, S.; Iliopoulos, N.; Zervakis, G.I. The effects of olives harvest period and production year on olive mill wastewater properties: evaluation of Pleurotus strains as bioindicators of the effluent's toxicity. Chemosphere 2013, 92, 399-405.

7. Zerva, A.; Zervakis, G.I.; Christakopoulos, P.; Topakas, E. Degradation of olive mill wastewater by the induced extracellular ligninolytic enzymes of two wood-rot fungi. J. Env. Manag. 2017, 203, 791-798.

8. Zerva, A.; Savvides, A.L.; Katsifas, E.A.; Karagouni, A.D.; Hatzinikolaou, D.G. Evaluation of Paecilomyces variotii potential in bioethanol production from lignocellulose through consolidated bioprocessing. Bioresour. Technol. 2014, 162, 294-299.

9. Miller, GL. Use of dinitrosalicylic acid reagent for determination of reducing sugar. Anal. Chem. 1959, 31, 426-428.

10. Zerva, A.; Pentari, C.; Grisel, S.; Berrin, J.-G.; Topakas, E. A new synergistic relationship between xylanactive LPMO and xylobiohydrolase to tackle recalcitrant xylan. Biotechnol. Biofuels 2020, 13, 142.

11. Zhang, H.; Yohe, T.; Huang, L.; Entwistle, S.; Wu, P.; Yang, Z.; Busk, P.; Xu, Y.; Yin, Y. dbCAN2: a meta server for automated carbohydrate-active enzyme annotation. Nucleic. Acids Res. 2018, 46, W95-W101

12. Lee, S.E.; Hwang, H.J.; Ha, J.S.; Jeong, H.S.; Kim, J.H. Screening of medicinal plant extracts for antioxidant activity. Life Sci. 2003, 73, 167-179.

13. Chaita, E.; Lambrinidis, G.; Cheimonidi, C.; Agalou, A.; Beis, D.; Trougakos, I.; Mikros, E.; Skaltsounis, A.L.; Aligiannis, N. Anti-Melanogenic Properties of Greek Plants. A Novel Depigmenting Agent from Morus alba Wood. Molecules 2017, 22, 514.

14. Castanera, R.; Pérez, G.; Omarini, A.; Alfaro, M.; Pisabarro, A.G.; Faraco, V.; Amore, A.; Ramírez, L. Transcriptional and Enzymatic Profiling of Pleurotus ostreatus Laccase Genes in Submerged and Solid-State Fermentation Cultures. Appl. Environ. Microbiol. 2012, 78, 4037-4045.

15. Villares, A.; Moreau, C.; Bennati-Granier, C.; Garajova, S.; Foucat, L.; Falourd, X.; Saake, B.; Berrin, J.G.; Cathala, B. Lytic polysaccharide monooxygenases disrupt the cellulose fibers structure. Sci Rep. 2017, 7, 40262.

Publisher's Note: MDPI stays neutral with regard to jurisdictional claims in published maps and institutional affiliations. 
( 2020 by the authors. Submitted for possible open access publication under the terms and conditions of the Creative Commons Attribution (CC BY) license (http://creativecommons.org/licenses/by/4.0/). 